\title{
WICKER Hans-Rudolf ; FIBBI Rosita et HAUG Werner (Dir.), Les migrations et la Suisse. Résultats du Programme national de recherche "Migrations et relations interculturelles »
}

Emmanuelle Le Texier

\section{(2) OpenEdition}

\section{Journals}

Édition électronique

URL : https://journals.openedition.org/remi/4066

DOI : 10.4000/remi.4066

ISSN : $1777-5418$

Éditeur

Université de Poitiers

Édition imprimée

Date de publication : 1 décembre 2004

Pagination : 190-194

ISBN : 2-911627-38-5

ISSN : 0765-0752

Référence électronique

Emmanuelle Le Texier, « WICKER Hans-Rudolf ; FIBBI Rosita et HAUG Werner (Dir.), Les migrations et la Suisse. Résultats du Programme national de recherche "Migrations et relations interculturelles » », Revue européenne des migrations internationales [En ligne], vol. 20 - n³ | 2004, mis en ligne le 25 septembre 2008, consulté le 16 avril 2022. URL : http://journals.openedition.org/remi/4066 ; DOI : https://doi.org/ 10.4000/remi.4066

Ce document a été généré automatiquement le 16 avril 2022.

(c) Université de Poitiers 


\title{
WICKER Hans-Rudolf ; FIBBI Rosita et HAUG Werner (Dir.), Les migrations et la Suisse. Résultats du Programme national de recherche "Migrations et relations interculturelles »
}

\author{
Emmanuelle Le Texier
}

\section{RÉFÉRENCE}

WICKER Hans-Rudolf ; FIBBI Rosita et HAUG Werner (Dir.), Les migrations et la Suisse. Résultats du Programme national de recherche « Migrations et relations interculturelles ». Zurich, Seismo, 2003, 566 p. ISBN: 3-908239-92-3

Les migrations et la Suisse constitue un ouvrage collectif de référence qui propose, d'une part, un état des lieux sur la place des immigrants et de la population issue de l'immigration dans la société suisse et, d'autre part, une réflexion sur le «modèle suisse » et les transformations des relations interculturelles liées à l'accueil des immigrants. Ce livre ambitieux reflète les résultats du Programme national de recherche 39 "Migrations et relations interculturelles " lancé en 1995 pour évaluer différents aspects de l'intégration des migrants depuis les années 1950. La Suisse présente la plus grande diversité culturelle, linguistique, religieuse et ethnique des pays européens : depuis 1945, deux millions d'individus sur les 7,2 millions d'habitants sont immigrants ou issus de l'immigration. Les flux sont d'abord arrivés d'Italie et d'Espagne jusque dans les années 1970. La migration économique a été ensuite progressivement remplacée par une migration familiale dans les années 1980, issue du 
Portugal, de la Turquie et de l'ex-République de Yougoslavie. Les réfugiés principalement d'Indochine, du Sri Lanka et du Moyen-Orient ont également contribué à la diversification des populations accueillies. Depuis les quinze dernières années, cette hétérogénéité s'est encore accrue avec des migrants venus d'Europe de l'Est notamment. Les politiques migratoires suisses semblent désormais similaires à celle de l'Allemagne ou des États-Unis, privilégiant trois principes essentiels : la nécessité de l'immigration (pour alimenter la croissance démographique et économique); la sélectivité accrue de migrants qualifiés; et le contrôle renforcé des frontières pour limiter l'immigration non qualifiée.

2 Le fil rouge qui a animé les recherches est clairement exposé dans l'introduction de Wicker. Les chercheurs se sont attachés non tant aux causes des migrations et aux contextes de départ, qu'à la manière dont "l'immigration touche des systèmes sociaux partiels et comment elle incite les institutions étatiques et sub-étatiques à agir » (p. 54). La question de l'intégration reste donc centrale, mais appréhendée à la lumière d'une approche qui considère les migrants comme des acteurs qui déploient logiques d'intérêts, ressources et stratégies propres. En outre, les écrits portent sur un segment spécifique des immigrants, délaissant les migrations transfrontalières pour se focaliser sur les enjeux sociaux de la migration dite asymétrique. Enfin, les contributions éclairent les potentiels de contrôle et d'action en matière de migration et d'intégration. Fort justement et en guise d'autocritique, Wicker souligne le risque d'un "effet synchronisateur » entre la communauté de chercheurs et des politiques qui diffusent des représentations de l'immigration non plus tant en termes de "perte de l'identité nationale", mais de "possible érosion de la solidarité sociale et étatique» du fait des migrations (p. 55). En filigrane, Wicker avance que si l'apport scientifique de l'ouvrage est indiscutable, sa portée politique demeure à double tranchant. Mais cette interrogation sur les enjeux de l'interprétation des résultats et de leur sens marque l'ensemble des contributions, ce qui leur attribue alors un caractère de réflexivité rarement présent dans cette littérature.

3 Il ne s'agit pas ici de rendre compte de l'ensemble des contributions, vingt et une au total, mais de mettre en lumière l'intérêt de cet ouvrage sous l'angle de la meilleure connaissance du phénomène des migrations en Suisse et de son apport comparatif et méthodologique pour les études migratoires. Quatre grandes thématiques organisent la réflexion menée.

4 La première partie touche aux enjeux de la mobilité transnationale, des droits civiques et des identités. Le changement sémantique, de la catégorie " étranger » et " réfugié » à celle de «migrants » dans les années 1980, marque en Suisse - et souvent ailleurs une mutation de la perception du phénomène de mobilité transnationale. Celle-ci naît de la prise en compte du contexte de départ et des liens permanents que tissent les migrants avec leur pays d'origine (l'entre deux), mais également au principe de mobilité (droit de rester, obligation de partir). En ce sens, la création de régimes d'immigration dans les pays européens implique des procédures de sélection et des mécanismes d'intégration plus ou moins libéraux.

5 La contribution de Mahnig et Piquet, La politique suisse d'immigration de 1948 à 1998 : évolution et effets, retrace bien les dynamiques et transformations des politiques migratoires, oscillant entre établissement de quotas et politique ouverte. Jusqu'à la Première Guerre mondiale, les processus de naturalisation étaient privilégiés pour assimiler les «étrangers». À cette période ont succédé jusqu'aux années 1980 des 
tentatives plus ou moins réussies d'établir des seuils pour restreindre l'accès des étrangers au territoire (création d'un office central pour la police des étrangers en 1917, loi fédérale restrictive en 1934, critères de naturalisation). La phase actuelle est celle qui passe de la dichotomie classique "travailleur immigré/citoyen" à celle de "désiré/non désiré ». Cette dynamique se note à la fois dans la sélection, voire la demande, d'une immigration qualifiée et dans l'interprétation restrictive de la politique d'asile, dont la décentralisation implique de surcroît un traitement non égalitaire. Ainsi, l'adoption de la législation sur les droits civiques en 1992 qui prévoit une naturalisation facilitée et reconnaît la double nationalité marque une ouverture, mais limitée aux citoyens d'Europe occidentale. Le double discours vise donc à mieux inclure les individus «culturellement proches» et à exclure les groupes «culturellement différents ».

6 La contribution de Giugni et Passy, Modèles de citoyenneté et mobilisation des immigrés en Suisse et en France. Une approche des opportunités politiques, est aussi très éclairante, du fait de l'approche comparative entre les modèles suisse et français de l'intégration. À partir d'une analyse systématique du contenu d'un quotidien national entre 1990 et 1998 (Neue Zürcher Zietung pour la Suisse et Le Monde pour la France), les revendications politiques des immigrés dans l'espace public ont pu être catégorisées selon leur objet: immigration et asile, incorporation, relations entre minorités et majorité, politique à l'égard du pays d'origine, etc. Pour les auteurs, le modèle d'intégration français, civique et républicain, incite les mobilisations des migrants dans la sphère publique pour la protection de leurs intérêts collectifs. À l'inverse, le modèle suisse, basé sur la ségrégation ethnique, favorise le maintien des liens au pays d'origine et les migrants disposent de peu de ressources symboliques, de légitimité ou d'opportunités politiques pour s'exprimer dans l'espace public. Cette différence trouve ses racines dans la mise en place des régimes d'immigration qui, en Suisse, se caractérisent par la difficulté de l'accès à la citoyenneté et auraient tendance à animer un repli sur la culture ou les traditions d'origine. Ce sont donc les modèles de citoyenneté qui «dessinent clairement une frontière entre le citoyen et l'immigré et définissent les pratiques d'incorporation des populations migrantes au sein de l'espace national " (p. 105). La contribution de Giugni et Passy est stimulante car elle apporte un éclairage nouveau sur l'appréhension de la contestation politique et l'intégration des migrants dans un modèle suisse qualifié dans ses dimensions culturelle et légale de ethno-assimilationiste. La Suisse est en effet pluraliste «à l'égard des cultures traditionnellement reconnues dans le cadre du fédéralisme et des procédures informelles d'intégration des minorités nationales, elle l'est nettement moins envers les minorités ethniques issues de l'immigration» (p. 111). La prise en compte des régimes migratoires et des opportunités politiques devient essentielle à la compréhension des modes d'intégration des migrants.

7 La deuxième partie de l'ouvrage s'intitule Les villes et leurs étrangers. Les trois contributions, héritières de la sociologie urbaine développée par l'École de Chicago, démontrent que la concentration des populations d'origine étrangère n'est assurément pas propre à la Suisse. En outre, la ségrégation résidentielle des populations immigrées ou issues de l'immigration n'est pas nouvelle : sa répartition géographique est même plutôt stable depuis la fin des années 1970 avec une forte présence dans les zones urbaines et une concentration dans les centres urbains. En revanche, la mutation importante est celle de la composition des immigrés. La proportion des "extraeuropéens» diminue en effet au profit des européens, et l'hétérogénéité s'est accrue. Ainsi, à Berne, Bâle et Zurich, la population est majoritairement d'origine étrangère, 
mais une nouvelle ligne de partage sépare les nouveaux migrants des anciens. Wimmer, dans Étrangers établis et outsiders indigènes. Formation de catégories sociales et réseaux de relations dans trois quartiers d'immigrants, propose une analyse des réseaux sociaux dans des quartiers de ces trois villes, afin de déterminer si la concentration spatiale et ethnique signifie isolement social ou peut impliquer au contraire interaction sociale. L'intérêt de cette approche est de mettre en avant l'établissement de catégories sociales plus en termes de notion d'ordre et de désordre, plutôt que d'étrangers et de citoyens. Ces formations évoluent en fonction de la nationalité d'origine et de la génération. Ainsi, il n'est pas tant important de savoir que «les Suisses nouent plutôt des relations avec les Italiens et les Européens du Nord, les Italiens de première génération avec des Suisses " (p. 223), que de penser l'endogamie comme une constante de l'ensemble des relations sociales. Il est alors possible d'appréhender simultanément l'existence d'enclaves ethniques et la formation de réseaux trans-ethniques. Il est regrettable toutefois que Wimmer n'inclut pas dans son analyse l'effet de période, les transformations du contexte de réception ou l'indicateur des mariages mixtes.

8 La troisième partie touche aux aspects de Migration et économie par le biais de cinq contributions, mêlant aspects économiques, juridiques, sociologiques et politologiques. C'est dans cette partie que la question de l'interprétation des résultats et du sens des recherches élaborées est la plus sensible. Toutes contribuent à une évaluation des effets induits par la migration sur les économies régionale et nationale de la Suisse. De nature très technique et sectorielle, ces chapitres se destinent à un lectorat plus spécialiste et n'élaborent pas de comparaison avec d'autres modèles. Les recherches portent sur la valeur attribuée à la main-d'œuvre étrangère, sur les logiques contradictoires de l'ouverture et de la fermeture du marché, sur les tensions qui peuvent affecter le marché du travail. Ainsi, un quart seulement des étrangers possède un emploi sur le marché du travail suisse et les réglementations différenciées - droit des étrangers et droit d'asile - complexifie la situation. Il est toutefois possible d'établir trois catégories de travailleurs: les étrangers au statut précaire - permis à l'année, frontaliers, saisonniers, résidant de courte durée - qui serviraient d'amortisseur conjoncturel ( $p$. 40) ; les demandeurs d'asile qui deviendraient les nouveaux Gastarbeiter ; et enfin les étrangers employés victimes de discrimination (à l'embauche ou au salaire). Flückiger et Ramirez dans Positions hiérarchiques et ségrégation par origine en Suisse, étudient ainsi le processus appliqué implicitement par les employeurs pour sélectionner les candidats à une promotion professionnelle. Par une méthode statistique sophistiquée qui permet de distinguer une composante discriminatoire et une composante expliquée par les caractéristiques individuelles des employés dans un indice global de ségrégation, ils concluent que «les positions hiérarchiques inférieures occupées par les étrangers s'expliquent en grande partie par le fait que les employeurs appliquent à leur encontre une politique discriminatoire 》 (p. 305). Mais le confinement des étrangers à des positions inférieures est aussi accentué par le manque général de formation et par le statut et type de permis de travail de la population étrangère.

9 Finalement, la dernière partie de l'ouvrage collectif, intitulée Migration et intégration à la société civile, forme avec la première partie la plus grande richesse du livre par un retour au processus d'intégration et une ouverture à des approches psychologiques et sociologiques. Centrées sur l'acteur individuel dans ses interactions aux institutions sociales (formation, social, santé, école), ces approches tentent d'analyser les transformations institutionnelles provoquées par le contact à des populations étrangères ou issues de l'immigration de plus en plus hétérogènes. Oscillant entre 
pratiques assimilationnistes et promotion du multiculturalisme, se débat la recherche d'un modèle différencié d'intégration (maintien de la langue maternelle comme facteur d'augmentation du capital social et économique, intégration de la différence culturelle dans les services de santé, approche pédagogique intégrant l'environnement social des enfants etc.).

10 Le retour sur le processus d'intégration permet aussi de distinguer trois formes de réponse des institutions helvétiques. Le modèle d'assimilation, à la base de la compréhension de l'intégration en Suisse, s'appuie sur un constat de déficit à combler (création de cours de soutien, de commissions pour les étrangers, etc.). Dans les années 1980, une seconde phase, pondérée par une certaine tolérance aux cultures étrangères, a été initiée. Les mutations de l'origine des flux migratoires, de plus en plus extraeuropéens ont renforcé la pluralisation ethnique, religieuse, linguistique et culturelle. L'approche multiculturaliste n'a pourtant pas pénétré dans les institutions, hormis dans le secteur psychosocial et médical. Deux études de cas, l'une de Testa-Mader, Clerici et Degrate, La population étrangère au Tessin: malaise psychique et utilisation des services psychiatriques publics; l'autre de Singy, Weber et Guex, Les migrants face au système de soins : une expérience à Lausanne, permettent d'approcher la relation médecin/ patient en terme asymétrique afin de prendre en compte les spécificités de la population migrante, en particulier des demandeurs d'asile. Cette phase ad hoc a laissé place depuis les années 1990 à une nouvelle étape marquée par la considération de l'intégration comme un objectif politique. Celui-ci se doit d'inclure toutes les institutions, non plus à partir d'un regard sur le "culturellement autre ", mais sur l'examen des ressources matérielles et immatérielles dont disposent les migrants pour s'intégrer.

11 À ce titre, la contribution de Bolzmann, Fibbi et Vial, Que sont-ils devenus? Le processus d'insertion des adultes issus de la migration, apporte une tonalité optimiste sur l'intégration de la deuxième génération, enfants d'immigrés italiens et espagnols. À l'encontre des thèses avancés aux États-Unis sur le déclin de la seconde génération ou l'assimilation segmentée (de la deuxième génération asiatique ou latino), les auteurs montrent les trajectoires de mobilité sociale et la formation d'identités culturelles particulières de générations qui vivent leur double appartenance culturelle avec réussite. L'approche quantitative et qualitative (402 entretiens réalisés avec des adultes de 18 à 35 ans issus de l'immigration et 203 avec des adultes nés de familles suisses) démontre qu'en matière de formation scolaire et professionnelle, d'intégration dans le marché du travail ou du chômage, il n'existe presque aucune différence entre enfants issus de familles suisses et enfants issus des familles italiennes ou espagnoles. Comparés aux études en France ou en Allemagne, plus proche du modèle d'assimilation segmentée, les résultats penchent pour une assimilation linéaire pour une génération préservée de la stigmatisation. Le mode d'incorporation des jeunes d'origine espagnole/italienne en Suisse se caractérise donc "par une intégration sociale réussie (formation, emploi), une assimilation sélective de certains traits de la culture locale (langues, valeurs d'achievement, etc.) et le maintien de certains traits de la culture d'origine (valeurs et pratiques familiales, ethos du travail » (p. 457).

Les Migrations et la Suisse est donc un ouvrage de référence pour le lecteur qui souhaite disposer d'une approche pluridisciplinaire des migrations en Suisse depuis les années 1950. Comme tout ouvrage collectif, il pêche parfois par une inégale qualité des contributions et un fil rouge qui se fait parfois difficile à saisir. Certains seront surpris 
de l'absence de traductions de citations en langue étrangère et de quelques inexactitudes relevées dans les tableaux statistiques, dont la pertinence n'est pas toujours explicitée. Mais l'effort de collaboration entre chercheurs de disciplines distinctes et la complétude d'approches scientifiques et politiques différenciées représente une véritable gageure. Les Migrations et la Suisse offre un état des lieux quasi exhaustif, dont seuls deux aspects font défaut: le caractère transnational des migrations (mobilisations collectives transnationales, rôle des pays d'origine dans les modifications éventuelles des politiques migratoires suisses, etc.); et les effets éventuels de l'européanisation des politiques migratoires et d'asile sur les institutions helvétiques. Enfin, l'apport de cet ouvrage à la compréhension des phénomènes migratoires hors du cadre suisse, soit par le biais méthodologique, soit par l'aspect comparatif, soit encore par la théorisation, constitue une indéniable réussite.

\section{AUTEURS}

\section{EMMANUELLE LE TEXIER}

Marie Curie Fellow, CEDEM 